\title{
Commentary
}

\section{What Is a Molecule?}

\section{Shu-Kun Lin}

Molecular Diversity Preservation International (MDPI), Saengergasse 25, CH-4054 Basel, Switzerland Tel.: +41 79322 3379, Fax: +41 61302 8918, E-mail: lin@ mdpi.org, URL: http://www.mdpi.org/lin/

\section{Dedicated to the memory of Linus Pauling}

Received: 1 October 1999 / Accepted: 11 October 1999 / Published: 1 January 2000

During recent four years when I edited the chemistry journal Molecules (www.mdpi.org/molecules/) and handled chemical samples at MDPI (http://www.mdpi.org), an international nonprofit organization for worldwide collection, deposit and exchange of chemical samples, I always had a question in mind: what is a molecule? Finally I would like to define it as a structure of atom assemblage having a decay constant $(\tau)$ longer than the resonance period $(\tau>1 / v$, where $v$ is the resonance [1] frequency). All molecules are decaying, because at least all the atoms are decaying as in turn all the sub-atomic particles are decaying, even though most of them have very long lifetime. All molecules have at least constant dynamic electronic motions which is described by Pauling as resonance that should be characterized by an oscillation frequency [2]. Therefore, a static molecular structure is defined as stable only in a relative sense. If it is shorter than that, we cannot study the molecule. We are interested in understanding the nature of the chemical bonds or the molecular structure and stability and many other properties under various conditions. The structure and property of individual molecules will be the main topic of molecular science.

The interaction and the collective behaviors of molecules will be another main topic. Molecules interact each other. They may recognize (molecular recognition), assemble, aggregate, etc. Macromolecules will have primary, secondary, tertiary and other supramolecular structures. The nature of these molecular interactions and molecular structures will be revealed with the continued hard work of physicists, chemists and biologists and scientists from other related fields of studies.

Physics, particularly quantum mechanics and statistical mechanics and possibly information theory can be used to guide our investigations. However, it is not true that now we already have some specific chemical theory which can resolve all or most of the outstanding problems in molecular sciences. Be-

(C) 2000 by Molecular Diversity Preservation International (MDPI, http://www.mdpi.org/) 
lieve it or not, the best and the most useful chemical bond theory is still Pauling's resonance theory [1].

Pauling is perhaps the greatest chemist (or molecular scientist) of the last century [3-5]. Through his research career, he was not only a master of theory, but also in experiments and in the close combination of the two. His theories will be continuously used and hopefully developed further in this century. His scientific attitude and methodology are taken as our heritage. He is also one of the greatest humanitarians of the last century. His conscience and social responsibility as a scientist are models for future scientists to follow.

At the end of his book [1], for instance, Pauling was still bothered by the problem of muscle contraction - a problem which is still outstanding today. Pauling attacked problems of many very diverse phenomena on the bases of molecular behavior. Thus, he can be said to have invented the subjects of molecular biology, molecular medicine and molecular disease.

Let us learn from Pauling and perform molecular science to the same standard as he would expect.

\section{References and Notes}

1. Pauling, L. The Nature of the Chemical Bond; 3rd ed.; Cornell University Press: Ithaca, 1960.

2. It might be an interesting research topic to define and calculate the resonance frequency.

3. Whether a theory is successful is judged by its application. Among many discoveries, the structural elucidation of protein and DNA was based on Pauling's theory.

4. Linus Pauling (28 February 1901 - 19 August 1994). Pauling's biographic summary is at the http://www.nobel.se/laureates/peace-1962-bio.html website. I have read two recent books of Pauling's biography. Neither book is prepared by a chemist.

5. Linus Pauling's life spanned the Twentieth Century at http://www.paulingexhibit.org/.

(C) 2000 by Molecular Diversity Preservation International (MDPI, http://www.mdpi.org/) 